\title{
Design principles and field performance of a solar spectral irradiance meter
}

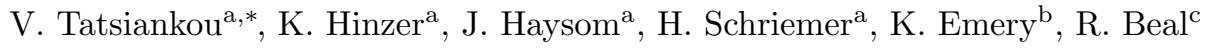 \\ ${ }^{a}$ University of Ottawa, SUNLAB, Ottawa, Ontario, K1N 6N5, Canada \\ ${ }^{b}$ National Renewable Energy Laboratory, Golden, Colorado, 80401, USA \\ ${ }^{c}$ COFOVO Energy Inc., Ottawa, Ontario, K1N 6N5, Canada
}

\begin{abstract}
A solar spectral irradiance meter (SSIM), designed for measuring the direct normal irradiance (DNI) in six wavelength bands, has been combined with models to determine key atmospheric transmittances and the resulting spectral irradiance distribution of DNI under all sky conditions. The design principles of the SSIM, implementation of a parameterized transmittance model, and field performance comparisons of modeled solar spectra with reference radiometer measurements are presented. Two SSIMs were tested and calibrated at the National Renewable Energy Laboratory (NREL) against four spectroradiometers and an absolute cavity radiometer. The SSIMs' DNI was on average within $1 \%$ of the DNI values reported by one of NREL's primary absolute cavity radiometers. An additional SSIM was installed at the SUNLAB Outdoor Test Facility in September 2014, with ongoing collection of environmental and spectral data. The SSIM's performance in Ottawa was compared against a commercial pyrheliometer and a spectroradiometer over an eight month study. The difference in integrated daily spectral irradiance between the SSIM and the ASD spectroradiometer was found to be less than $1 \%$. The cumulative energy density collected by the SSIM over this duration agreed with that measured by an Eppley model NIP pyrheliometer to within $0.5 \%$. No degradation was observed.
\end{abstract}

Keywords: solar spectral irradiance meter, SSIM, direct normal spectral irradiance, solar resource assessment, atmospheric parameterization, spectroradiometer, absolute cavity radiometer, pyrheliometer, solar spectrum

\section{Introduction}

Photovoltaic (PV) system performance fundamentally depends on the incident solar spectrum, which fluctuates both temporally and geographically. Different solar technologies respond to such fluctuations in varying degree, with

\footnotetext{
*Corresponding author: Tel +(1) 613-413-1877

Email address: viktar.tatsiankou@gmail.com (V. Tatsiankou)
}

Preprint submitted to Solar Energy

February 28, 2016 


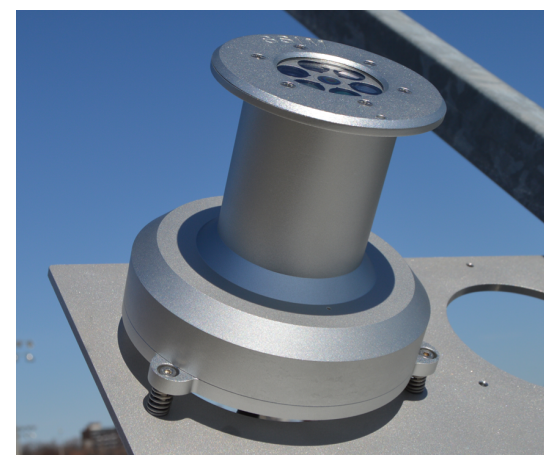

(a)

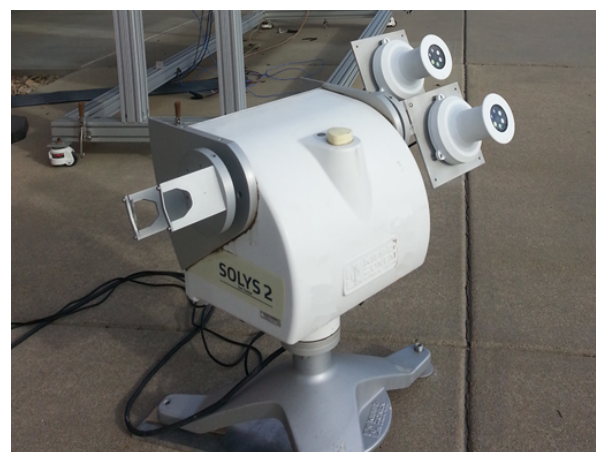

(b)

Figure 1: (a) SSIM installed at the University of Ottawa solar test site. (b) Two SSIMs tested at the NREL's Outdoor Test Facility.

single junction devices typically less influenced by spectral changes than are multi-junction solar cells (MJSC). A MJSC, used terrestrially in concentrator photovoltaic systems, consists of series-connected subcells with different bandgaps for more efficient use of the solar spectrum than is possible with a single junction because thermalization and transmission losses are reduced. In such a structure, the lowest current-generating subcell limits the performance of other subcells, so for optimal performance a MJSC is designed to be near current matched for a specific spectrum, such as the AM1.5D from the ASTM G173 standard (McMathon et al. 2002 Guter et al., 2009: Wiemer et al., 2011). But under outdoor conditions the solar spectrum deviates from the reference due to varying meteorological conditions, resulting in subcell current mismatch and thus reduced concentrator photovoltaic system performance (Muller et al., 2010 . Chan et al., 2014, García-Domingo et al., 2014). Quantification of the solar spectrum is thus required for a complete performance analysis of these systems.

A field spectroradiometer is typically the most accurate way to determine a local solar spectrum. However, these measurements are rarely performed due to prohibitive instrumentation costs, which can approach $\$ 100 \mathrm{~K}$ (depending on spectral range and manufacturer). The more cost-effective pyrheliometer, which measures the broadband direct normal irradiance (DNI) with an estimated uncertainty of $\pm 2 \%$ (Sengupta et al., 2015), is typically used instead, but it does not provide the desired spectral information.

The solar spectrum may be reconstructed using modeling tools if sufficient local data exists. The Simple Model of the Atmospheric Radiative Transfer of Sunshine (SMARTS) has become the standard modeling tool (Gueymard, 1995. 2001). However, SMARTS requires inputs, such as the aerosol optical depth (at $500 \mathrm{~nm}$ ), precipitable water vapor content, and ozone column amount, that are not often known locally. The Aerosol Robotic Network (AERONET), with over 300 stations worldwide, can provide these parameters to nearby sites (Holben et al., 1998, 2001) or can be used to interpolate necessary local parameters (Chan 
et al., 2013), but the AERONET site sparsity remains a severe constraint on the general applicability and accuracy of this technique. By contrast, we use an instrumentation-based approach for local spectral reconstruction via limited spectral sampling that retains the accuracy of the spectroradiometer but at a far lower price point. Historically, this technique for derivation of the continuous spectral distribution of sunlight was attempted before (Michalsky and Kleckner. 1984 Osterwald et al., 1988). More specifically, NREL developed the Atmospheric Optical Calibration System (Cannon and Hulstrom, 1993) as a low-cost measurement for spectral solar irradiance based on a SPCTRL2 model (Bird and Riordan, 1986) and a four-channel sun photometer.

Our solar spectral irradiance meter (SSIM), shown in Fig. 1a, uses groundbased measurements to inform software algorithm for rapid resolution of the location-specific solar spectrum across the entire 280-4000 $\mathrm{nm}$ range. The numerical integration of the SSIM's spectrum yields the DNI as typically measured by a pyrheliometer. It is a cost-effective alternative to purchasing a spectroradiometer and a pyrheliometer. The irradiance is measured in six carefully chosen wavelength bands, using low-cost silicon photodiodes with bandpass filters, to allow spectral reconstruction through parametrization of the major atmospheric processes (Tatsiankou et al., 2013).

In 2013, a two month deployment at the SUNLAB Outdoor Test Facility showed encouraging results, with the SSIM and Eppley pyrheliometer DNI agreeing within $\pm 1.5 \%$, on average (Tatsiankou et al., 2014: Tatsiankou, 2014). Good spectral agreement between the SSIM and a commercial spectroradiometer was also observed.

Below we describe the design features of our commercially packaged SSIMs, and the parameterized transmittance model which the spectral reconstruction algorithm implements. Calibration and comparison results against National Renewable Energy Laboratory (NREL) multiple spectroradiometers and one of their primary absolute cavity radiometers are presented, followed by an eight month comparative study at the SUNLAB Outdoor Test Facility with an Eppley model NIP pyrheliometer and an ASD FieldSpec 3 spectroradiometer.

\section{SSIM design features}

The SSIM uses silicon photodiodes coupled with six bandpass filters to measure the spectral DNI in six wavelength bands with $10 \mathrm{~nm}$ full widths at half maximum (FWHM). By sampling the solar spectrum in these carefully selected

Table 1: SSIM's channel specifications and target atmospheric constituents Tatsiankou et al. 2013)

\begin{tabular}{ccc}
\hline Channel centre $(\mathrm{nm})$ & Target parameter & FWHM $(\mathrm{nm})$ \\
\hline $420,500,780,1020$ & Aerosols & 10 \\
610 & Ozone & 10 \\
940 & Water vapor & 10 \\
\hline
\end{tabular}


wavelength channels, we are able to quantify aerosol absorption and scattering, ozone absorption, and water vapor absorption - three of the six dominant atmospheric processes that parameterize the spectrum. Table 1 shows which wavelength channels target each parameter. This is possible because the SSIM's channels are chosen in spectral regions where only one unknown atmospheric constituent dominates. For example, channels centered at 500 and $780 \mathrm{~nm}$ can be used to deduce aerosol extinction for every wavelength in between these bands, since they are not significantly affected by either ozone or water vapor absorptions (Tatsiankou et al. 2013$)$. The impact of the three remaining processes - mixed gas $\left(\mathrm{O}_{2}, \mathrm{CO}_{2}\right.$, and $\left.\mathrm{CH}_{4}\right)$ absorption, $\mathrm{NO}_{2}$ absorption, and Rayleigh scattering - on spectral composition are primarily determined from local temperature and air pressure.

Each photodiode is situated within a collimation tube whose aperture and field stop geometries conform to the World Meteorological Organization standard for radiometric measurements of DNI (WMO, 2008). The $5^{\circ}$ field of view is achieved by the geometric design of the SSIM's collimation tubes, which are concentric with the photodiodes. The aperture of each collimation tube is covered by a bandpass filter and is protected from the ambient environment by a BK-7 front window. The instrument does not require temperature control, because it applies an automated temperature calibration for each photodiode. The internal humidity is kept at a low level with a desiccant. The anodized aluminum housing encloses a data acquisition printed circuit that sequentially measures photodiode current from each channel, as well as ambient temperature and pressure. The photodiode current measuring circuit features a $0.1 \mathrm{pA}$ input bias and $0.01 \mathrm{mV}$ input offset transimpedance amplifier coupled with a 22-bit delta-sigma analog-to-digital converter. The total power consumption of the circuit is approximately $0.7 \mathrm{~W}$. The photodiode current, ambient temperature and pressure data are sent via RS-485 communication protocol to a remote computer where specialized software driven by a graphical user interface implements spectral reconstruction over the $280-4000 \mathrm{~nm}$ range in real time (Tatsiankou et al. 2015). The reconstruction algorithm is based on the parameterized transmittance model discussed below.

\section{Parameterized transmittance model}

On a clear day the spectral DNI can be written as

$$
\begin{array}{r}
S(\lambda)=S_{0}(\lambda) \cdot r^{2} \cdot T_{\mathrm{a}}(\lambda) \cdot T_{\mathrm{g}}(\lambda) \cdot T_{\mathrm{n}}(\lambda) \cdot T_{\mathrm{o}}(\lambda) . \\
T_{\mathrm{R}}(\lambda) \cdot T_{\mathrm{w}}(\lambda),
\end{array}
$$

where $S_{0}(\lambda)$ is the extraterrestrial irradiance (known as AM0), $r$ is the ratio of the average to actual Sun to Earth distance, and $T_{\mathrm{i}}(\lambda)$ is the transmittance of each atmospheric constituent, where the subscripts "a", "g", "n", "o", "R", and "w" referring to aerosol absorption and scattering, mixed gas absorption $\left(\mathrm{O}_{2}\right.$, $\mathrm{CO}_{2}$, and $\left.\mathrm{CH}_{4}\right), \mathrm{NO}_{2}$ absorption, ozone absorption, Rayleigh scattering, and water vapor absorption, respectively. The AM0 spectrum, with an integrated 
irradiance value of $1366.1 \mathrm{~W} / \mathrm{m}^{2}$, is adopted from the most recent measurements (Gueymard, 2004).

Eq. (1) can be expressed equivalently by Beer's law as

$$
\begin{aligned}
S(\lambda)=S_{0}(\lambda) \cdot r^{2} \cdot \exp [ & -\tau_{\mathrm{a}}(\lambda) \cdot m_{\mathrm{a}}-\tau_{\mathrm{g}}(\lambda) \cdot m_{\mathrm{g}}-\tau_{\mathrm{n}}(\lambda) \cdot m_{\mathrm{n}} \\
& \left.-\tau_{\mathrm{o}}(\lambda) \cdot m_{\mathrm{o}}-\tau_{\mathrm{R}}(\lambda) \cdot m_{\mathrm{R}}-\tau_{\mathrm{w}}(\lambda) \cdot m_{\mathrm{w}}^{*}\right],
\end{aligned}
$$

where $\tau_{i}$ and $m_{i}$ are the optical depth and the air mass associated with each atmospheric component, respectively, and each transmittance is given by

$$
T_{i}(\lambda)=\exp \left(-\tau_{i} \cdot m_{i}\right) .
$$

The optical depth is a dimensionless measure of atmospheric transparency, as influenced by absorption and scattering. The air mass values are functions of the zenith angle of the sun. The air mass of each component can be written as (Gueymard, 2006)

$$
m_{i}=\left[\cos (Z)+\kappa_{i 1} \cdot Z^{\kappa_{i 2}} \cdot\left(\kappa_{i 3}-Z\right)^{\kappa_{i 4}}\right]^{-1},
$$

where $Z$, zenith angle (in degrees) of the sun, calculated with the NREL solar position algorithm with an accuracy of $\pm 0.0003^{\circ}($ Reda and Andreas, 2004). The $\kappa_{i j}$ parameters are given in Table 2 (Gueymard, 2006).

The optical depths for each parameter depend uniquely on wavelength, thus allowing unambiguous spectral reconstruction from a very limited data set. Aerosol extinction can be modelled empirically within each region $y$ by Ångström's power law as (Ångström, 1929)

$$
\tau_{\mathrm{a}}(\lambda)=\beta_{\mathrm{y}} \cdot \lambda^{-\alpha_{\mathrm{y}}},
$$

where $\beta_{\mathrm{y}}$ and $\alpha_{\mathrm{y}}$ are coefficients that are determined experimentally. The SSIM has four aerosol channels, with each set of $\beta_{\mathrm{y}}$ and $\alpha_{\mathrm{y}}$ coefficients found and applied to the entire spectral region $y$ between two adjacent aerosol channels.

Optical depth due to mixed gases is caused by the cumulative effect of multiple gases that are assumed to be uniformly distributed in the atmosphere and is represented as

$$
\tau_{\mathrm{g}}(\lambda)=p_{\mathrm{CH}_{4}} \cdot A_{\mathrm{CH}_{4}}(\lambda)+p_{\mathrm{CO}_{2}} \cdot A_{\mathrm{CO}_{2}}(\lambda)+p_{\mathrm{O}_{2}} \cdot A_{\mathrm{O}_{2}}(\lambda),
$$

Table 2: Optical air mass coefficients for Eq. 3

\begin{tabular}{cccccc}
\hline Air mass & $i$ & $\kappa_{i 1}$ & $\kappa_{i 2}$ & $\kappa_{i 3}$ & $\kappa_{i 4}$ \\
\hline$m_{\mathrm{a}}$ & 1 & 0.169 & 0.182 & 95.318 & -1.954 \\
$m_{\mathrm{g}}$ & 2 & 0.484 & 0.0959 & 96.741 & -1.754 \\
$m_{\mathrm{n}}$ & 3 & 1.121 & 1.613 & 111.55 & -3.263 \\
$m_{\mathrm{o}}$ & 4 & 1.065 & 0.638 & 101.8 & -2.269 \\
$m_{\mathrm{R}}$ & 5 & 0.484 & 0.0959 & 96.741 & -1.754 \\
$m_{\mathrm{w}}^{*}$ & 6 & 0.107 & 0.114 & 93.781 & -1.920 \\
\hline
\end{tabular}

Note: $m_{\mathrm{w}}^{*}=m_{\mathrm{w}}^{0.943}$ 
Table 3: Mixed gases volume mixing ratios (Jacobson, 2005) with pressure and temperature correction coefficients (Gueymard, 2006)

\begin{tabular}{cccc}
\hline Mixed gas & $\chi_{\mathrm{g}}(\mathrm{ppmv})$ & $\gamma$ & $\varphi$ \\
\hline $\mathrm{CH}_{4}$ & 1.8 & 1.125 & 0.0473 \\
$\mathrm{CO}_{2}$ & 375 & 1 & 0 \\
$\mathrm{O}_{2}$ & 209500 & 1 & 0 \\
\hline
\end{tabular}

where $p_{i}$ is the path length (or the total column abundance) of the constituent gas (in atm-cm), and $A_{i}(\lambda)$ is its wavelength-dependent absorption coefficient (in $\mathrm{cm}^{-1}$ ). Each total column abundance depends on temperature and pressure as (Gueymard, 2006)

$$
p_{i}=\chi_{\mathrm{g}} \cdot H \cdot P_{\mathrm{r}}^{\gamma} \cdot T_{\mathrm{r}}^{\varphi},
$$

where $\chi_{\mathrm{g}}$ is the known volume mixing ratio of gas (in parts per million by volume), $H=8.429 \times 10^{5} \mathrm{~cm}$ is the scale height of the isothermal atmosphere, whose reference temperature and pressure are $288 \mathrm{~K}$ and $101.325 \mathrm{kPa}$, respectively; $P_{\mathrm{r}}$ and $T_{\mathrm{r}}$ are ratios of actual to reference pressure and temperature, respectively; and $\gamma, \varphi$ are pressure and temperature correction coefficients, respectively. Table 3 presents the volume mixing ratios along with pressure and temperature correction coefficients for mixed gases. This optical depth may therefore be calculated directly from the measured ambient temperature and pressure.

Ozone and nitrogen dioxide optical depths $\tau_{i}$, where $i=$ "o" or "n", can both be expressed as (Gueymard, 2006)

$$
\tau_{\mathrm{i}}(\lambda)=p_{\mathrm{i}} \cdot A_{\mathrm{i}}(\lambda) \cdot C_{\mathrm{i}}(\lambda)
$$

where $A_{\mathrm{i}}(\lambda)$ is an absorption coefficient in $\left(\mathrm{cm}^{-1}\right), C_{\mathrm{i}}(\lambda)$ is a unitless temperature correction function, and $p_{\mathrm{i}}$ is a path length (in atm-cm). $A_{\mathrm{i}}(\lambda)$ and $C_{\mathrm{i}}(\lambda)$ are known quantities for both ozone and nitrogen dioxide. While the ozone optical depth may be extracted from the SSIM ozone channel measurement, the nitrogen dioxide optical depth may be calculated directly. A total column abundance $p_{\mathrm{n}}=0.00118 \mathrm{~atm}-\mathrm{cm}$ is used, which is the average concentration of nitrogen dioxide in Montreal, Canada in 2005 under the assumption that this pollution layer is $1 \mathrm{~km}$ thick (Gilbert et al., 2005).

Rayleigh scattering is a well-known phenomenon (Bucholtz, 1995; Bodhaine et al. 1999) that allow the optical depth to be calculated from (Gueymard, 2006)

$$
\tau_{\mathrm{R}}(\lambda)=\frac{P_{\mathrm{r}}}{n_{1} \cdot \lambda^{4}+n_{2} \cdot \lambda^{2}+n_{3}+n_{4} \cdot \lambda^{-2}},
$$

where $P_{\mathrm{r}}$ is the ratio of site to sea level ambient pressure, and $n_{1}=117.341 \mathrm{~nm}^{-4}$, $n_{2}=1.511 \mathrm{~nm}^{-2}, n_{3}=0.0175$, and $n_{4}=8.774 \mathrm{~nm}^{2}$. 2006

The expression for precipitable water vapor optical depth is Gueymard,

$$
\tau_{\mathrm{w}}(\lambda)=p_{\mathrm{w}}^{0.943} \cdot \varepsilon_{1}(\lambda) \cdot \varepsilon_{2}(\lambda) \cdot A_{\mathrm{w}}(\lambda),
$$


where $A_{\mathrm{w}}(\lambda)$ is the known absorption coefficient $\left(\mathrm{cm}^{-1}\right), \varepsilon_{1}(\lambda)$ is a pressure scaling factor that accounts for water vapor's inhomogeneity in the atmosphere, $\varepsilon_{2}(\lambda)$ is a correction factor that improves parameterization away from the band centre under fluctuating humidity conditions, and $p_{\mathrm{w}}$ is the water vapor path length (in atm-cm). Absorption coefficients and correction factors for all atmospheric constituents are used as in SMARTS, except for some minor modifications. With the model definitely described, we now discuss its implementation in our spectral reconstruction algorithm.

\section{Algorithm implementation}

As seen in section 3, parameters $T_{\mathrm{g}}(\lambda), T_{\mathrm{n}}(\lambda)$, and $T_{\mathrm{R}}(\lambda)$ in Eq. (1) are directly calculable if geographic parameters, time stamp, ambient temperature, and site pressure are known. We now describe the algorithm used by the SSIM software to determine the three remaining transmittances, focusing primarily on the aerosols.

The spectral irradiance measured by the SSIM aerosol channel centred at $\lambda_{\mathrm{c}}$ is given by Eq. (1), except that $T_{\mathrm{o}}(\lambda)$ and $T_{\mathrm{w}}(\lambda)$ are set to unity for $\lambda_{1} \leq \lambda \leq \lambda_{2}$, where $\lambda_{1}$ and $\lambda_{2}$ delimit the $50 \mathrm{~nm}$ range of wavelengths for which the filter transmittance $F_{i}(\lambda)$ is nonzero. The manufacturer's generic filter transmittance data is used for each channel. Therefore, the simulated current of the photodiode for aerosol channel $i$ can be expressed as

$$
I_{i}=D \cdot r^{2} \cdot \int_{\lambda_{1}}^{\lambda_{2}} T_{\mathrm{a}}(\lambda) \cdot C_{i}(\lambda) \cdot d \lambda
$$

where $D$ is the active area of the photodiode, $r$ is the ratio of the average to actual Sun to Earth distance, $C_{i}(\lambda)=S_{0}(\lambda) \cdot T_{\mathrm{g}}(\lambda) \cdot T_{\mathrm{n}}(\lambda) \cdot T_{\mathrm{R}}(\lambda) \cdot F_{i}(\lambda) \cdot R(\lambda)$, and $R(\lambda)$ is the manufacturer's responsivity of the photodiode. The uniform trapezoidal rule is used to do the integration in Eq. (11), once $T_{\mathrm{a}}(\lambda)$ is known. Eqs. 2 and 5 in Eq. 3 gives

$$
T_{\mathrm{a}}(\lambda)=\exp \left[-\beta_{\mathrm{y}} \cdot \lambda^{-\alpha_{\mathrm{y}}} \cdot m_{\mathrm{a}}\right],
$$

and for a given aerosol extinction region $y$, the values of $\alpha_{\mathrm{y}}$ and $\beta_{\mathrm{y}}$ are optimized such that the simulated photodiode current agrees with the measured current from the SSIM. A Golden section search algorithm is used to find the values of $\alpha_{\mathrm{y}}$ and $\beta_{\mathrm{y}}$ that produce the smallest residual error (Press et al., 1992). This optimization routine is performed for each aerosol region $y$ to determine the aerosol transmittance $T_{\mathrm{a}}(\lambda)$. Calculation of the aerosol transmittance is a crucial step in deriving ozone and water vapor transmittances.

The spectral irradiance for ozone and water vapor channels is also given by Eq. (1). To determine $T_{\mathrm{o}}(\lambda)$, one sets $T_{\mathrm{w}}(\lambda)$ to unity, and vice-versa, using equations of the same form as Eq. (11) to find anticipated photodiode currents. The same optimization process is followed. The unknown ozone and water vapor transmittances are each expressed in like manner to Eq. 12. Both ozone 
and water vapor total column abundances, $p_{\mathrm{o}}$ and $p_{\mathrm{w}}$, are found with a Golden section search algorithm that minimizes the residual difference between the simulated and measured current values. Once all parameters are found, the final spectral distribution is calculated from Eq. (1) with a $1 \mathrm{~nm}$ resolution.

\section{Calibration and initial evaluation}

\subsection{Calibration}

A side-by-side on-sun comparison of the SSIM with a calibrated direct normal spectroradiometer was done. This test was performed at NREL's Outdoor Test Facility in Golden, Colorado, USA $\left(39.74^{\circ} \mathrm{N}, 105.18^{\circ} \mathrm{W}\right)$ on 17 November 2014. Two SSIMs (serial numbers SN102 and SN103) were mounted on a SOLYS 2 sun tracker, as shown in Fig. 11p. Data were acquired and processed from both devices every five seconds. The reference spectroradiometer, a Prede PGS-100, was located at NREL's Solar Radiation Research Laboratory, $200 \mathrm{~m}$ west and $100 \mathrm{~m}$ higher than the SSIM location. It has a silicon CCD array with a 1 degree field of view, a spectral range from 350 to $1050 \mathrm{~nm}$, a spectral bandwidth of $3.6 \mathrm{~nm}$, and a $0.7 \mathrm{~nm}$ resolution. Last calibrated on 6 November 2014 (mere days before this work), the PGS-100 spectroradiometer has traceability to the NIST Spectral Irradiance Lamp Standard through a LICOR LI-1800 secondary standard spectroradiometer.

The calibration procedure ensured that the SSIM's spectral irradiance in its six optical channels matched the irradiance from the PGS-100 at the corresponding wavelengths. Upon completion, six calibration factors were obtained, one for each channel, to compensate for deviations from the generic manufacturer's filter transmittances and photodiode responsivities used in our model. Immediately after calibration, the solar spectra from both SSIMs, the PGS100, and the extended range spectroradiometer combination EKO MS-710 and EKO MS-712 (WISER) were acquired, as shown in Fig. 2a. Both SSIMs are in excellent agreement with the PGS-100, which validates the SSIM design and parameterized transmittance model. As expected, the PGS-100 and WISER spectra agree to within the measurement uncertainties of both instruments.

\subsection{Short term performance}

Following the calibration procedure, the SSIM performance was extensively tested against NREL reference instrumentation. On 21 November 2014, four spectroradiometers, calibrated at NREL within the last 6 months using a calibrated standard lamp purchased from NIST, were used for a comparative analysis with the SSIMs. To the aforementioned PGS-100 and WISER, LICOR LI-1800 and ASDi FieldSpec 3 (ASD) spectroradiometers were added. The latter two instruments were mounted on a separate tracker within a few meters of the SSIMs. Excellent agreement was found between all instruments with their integral of spectral irradiance agreeing to within $1 \%$ as compared to the SSIMs' integral of spectral irradiance in corresponding wavelengths range, as shown in Fig. 2 $\mathrm{p}$. The ASD tends to slightly underestimate the solar irradiance for 

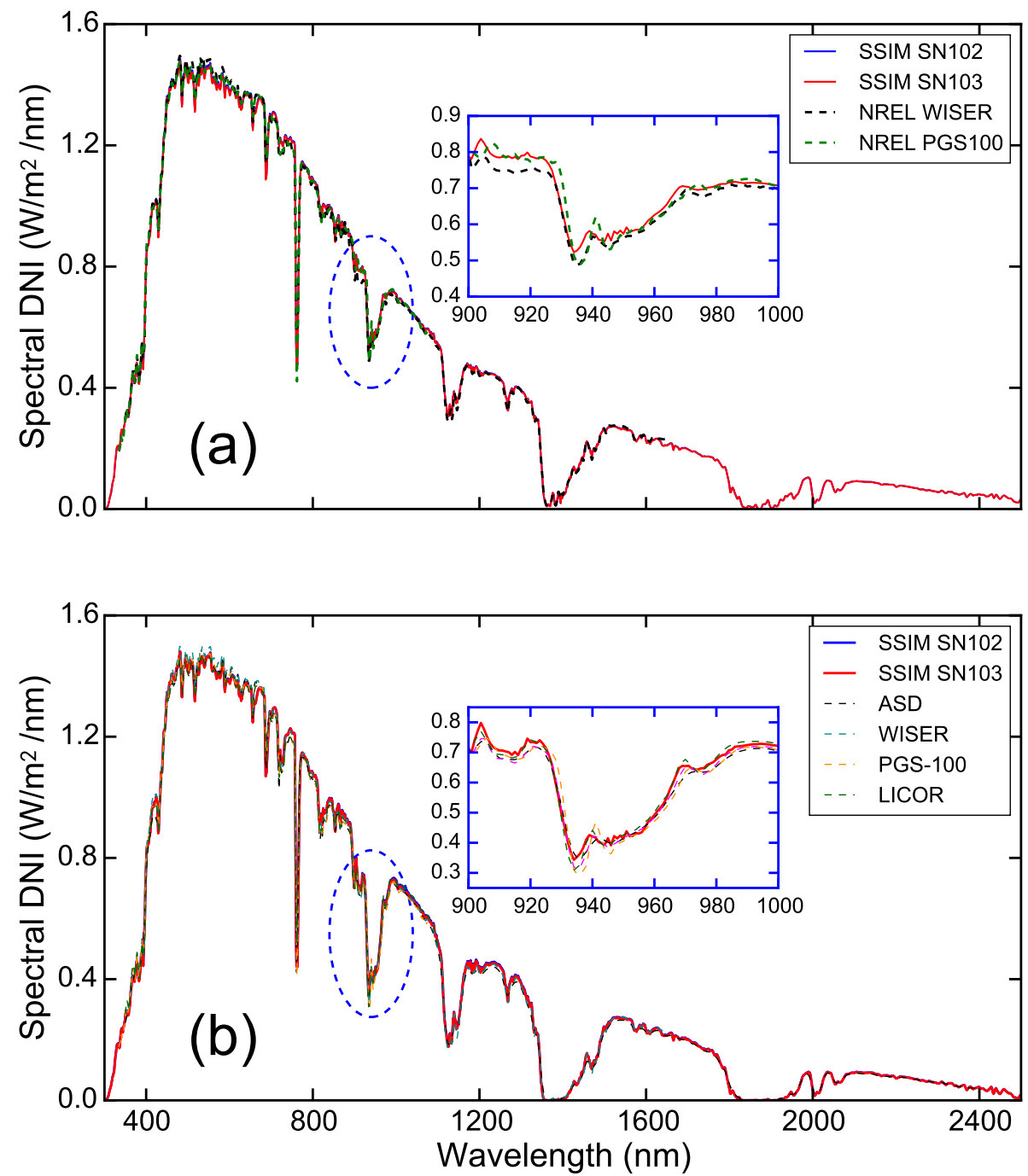

Figure 2: (a) Solar spectra measured by the SSIMs as compared to that measured by PGS-100 and WISER spectroradiometers immidiately after the calibration procedure on November 17 , 2014 at 13:05 MST. (b) Solar spectra measured by the SSIMs as compared to that measured by PGS-100, WISER, ASD, and LICOR spectroradiometers four days after the calibration on November 21, 2014 at 10:00 MST. Insets are magnifications around the water vapor channel to facilitate comparisons.

wavelengths below $450 \mathrm{~nm}$ as compared to the other reference instruments. The ASD's ability to measure the solar spectrum up to $2500 \mathrm{~nm}$, however, validates the SSIM performance in the IR range.

An Eppley HF absolute cavity radiometer (ACR) with traceability to the World Radiometric Reference was used to infer SSIM performance across the so- 

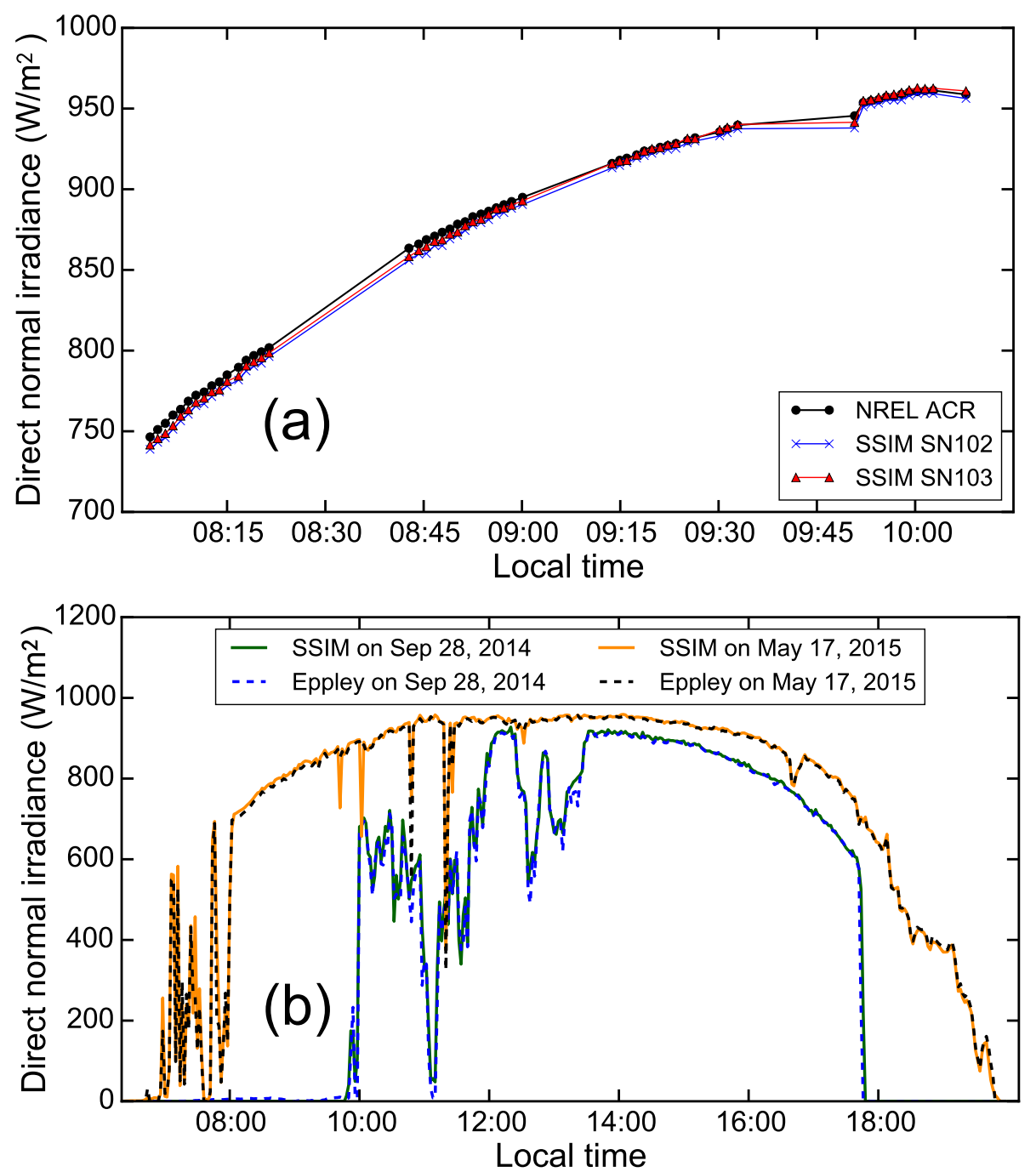

Figure 3: (a) Comparison of the DNI from the SSIMs SN102 and SN103 and the Eppley absolute cavity radiometer (ACR) on 21 November 2014. Data were acquired only under optimal atmospheric conditions. (b) Comparison of the DNI between the Eppley pyrheliometer and SSIM on a partially cloudy and a mostly clear sky day, occurring on 28 September 2014 and 21 May 2015, respectively.

lar spectral range through continuous measurement of the DNI (Fröhlich, 1991 , Reda, 1996). To compare with ACR DNI, SSIM DNI was obtained every five seconds and the reconstructed solar spectra were integrated across the entire 280-4000 nm range; the results are presented in Fig. 3a. Gaps appear in the measurement record since data acquisition was done only under clear sky conditions during which the sun's disk was unobscured by visible clouds. The DNI 

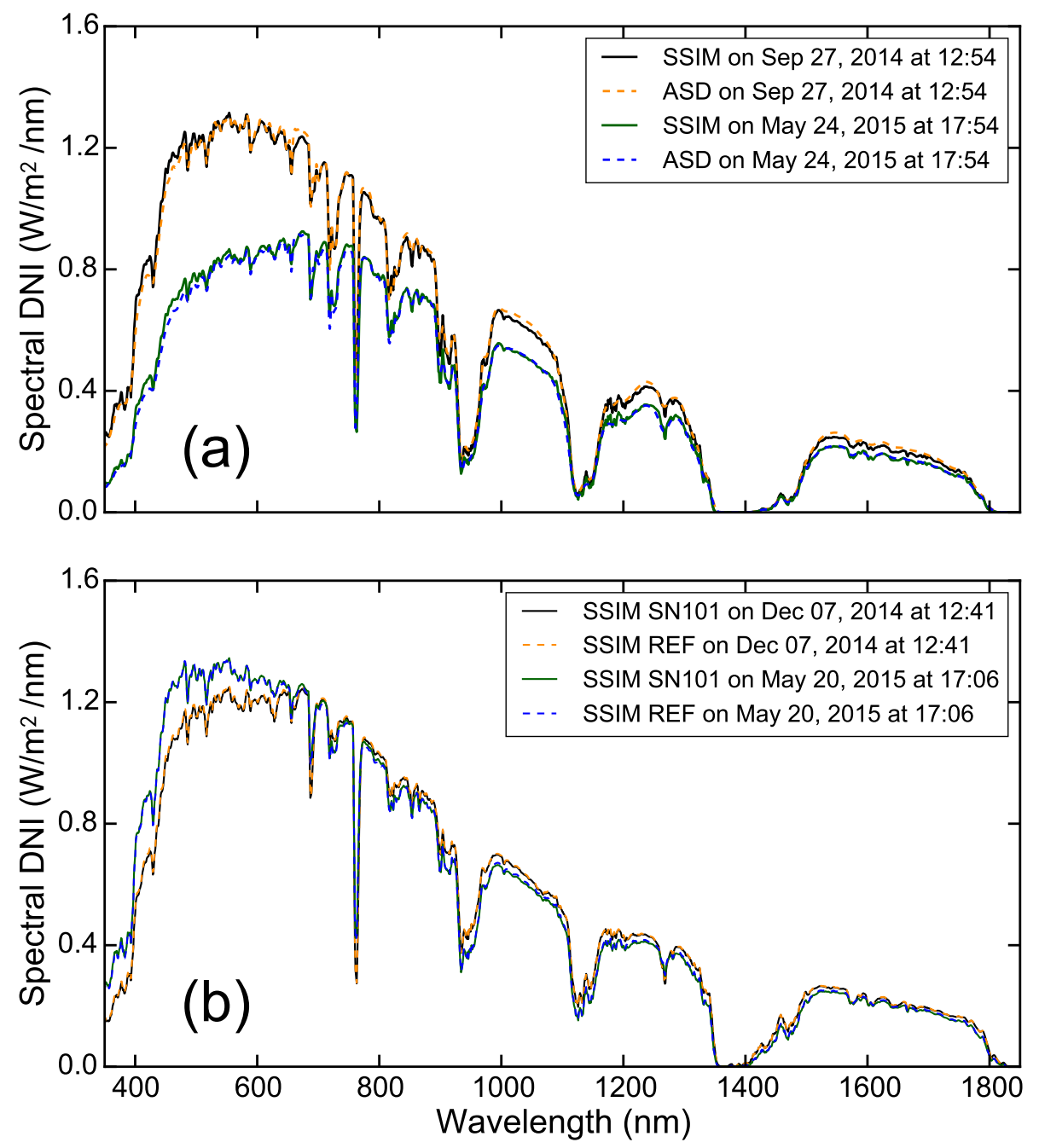

Figure 4: (a) Comparison of ASD spectroradiometer and SSIM spectra for selected timestamps. (b) Comparison of spectra as measured by SSIM SN101 and reference SSIM SN102 on the calibration date of 7 December 2014 and four months later on May 32015.

from both SSIMs was found to be within $\pm 1 \%$, on average, of ACR DNI. Since the ACR has a field accuracy of $0.5 \%$ (Michalsky et al., 2011), this is an exceptionally good agreement. These two SSIMs are now used as SUNLAB tertiary calibration standards.

\section{Seasonal field trials}

SSIM with a serial number SN101 was installed at the SUNLAB Outdoor Test Facility $\left(45.42^{\circ} \mathrm{N}, 75.68^{\circ} \mathrm{W}\right)$ in September 2014 . The device was situated 
on the same mechanical plate assembly as an Eppley model NIP pyrheliometer and an ASD FieldSpec 3 spectroradiometer, which measures spectral DNI in the 350-1830 nm range. The assembly was mounted on a Golden Sun GS-3500 dualaxis tracking system to point these instruments directly at the sun to within accuracy of $\pm 0.2^{\circ}$ (Schriemer et al. 2015).

SSIM SN101 was initially calibrated against the ASD spectroradiometer through direct on-sun comparison, using the same approach as at NREL. It was re-calibrated on 7 December 2014 against the NREL-calibrated reference SSIM SN102 and the new calibration file was also applied to all preceding data gathered by SSIM SN101.

The performance of the SSIM was evaluated against the Eppley pyrheliometer and the ASD spectroradiometer at the SUNLAB Outdoor Test Facility during the course of eight months. Representative comparisons of SSIM and pyrheliometer DNI profiles are shown in Figure 30 for two separate days, a partially cloudy on 28 September 2014, and a mainly clear on 17 May 2015. SSIM and pyrheliometer DNI were found to agree within $\pm 1 \%$ under clear sky conditions, on average. This exceptional agreement was only slightly degraded during times of rapid cloud transients due to the difference in detector response times. Similarly, representative comparisons of SSIM and ASD spectra shown in Fig. 4a for selected timestamps on 27 September 2014 and 24 May 2015, demonstrate a good spectral agreement between both instruments. The difference in the integrated spectral irradiance was found to be less than $1 \%$, on average, although the ASD spectroradiometer again tended to systematically underestimate the solar spectrum below $500 \mathrm{~nm}$. In service degradation of SSIM SN101 spectral channels was also periodically assessed over the eight month field trial by comparison with the SSIM calibration standard (SN102, stored indoors). SSIM spectra taken on 3 April 2015, about four months after the 7 December 2014 calibration date, show no appreciable difference between the in field unit and the reference standard, suggesting long term SSIM reliability.

To further validate SSIM longterm reliability, its cumulative and daily energy densities derived from the measured local solar spectra were compared to the corresponding measurements from the pyrheliometer across a continuous eight month period from 27 September 2014 to 28 May 2015. Data were acquired at two minute intervals and energy densities calculated for all values exceeding a DNI threshold of $50 \mathrm{~W} / \mathrm{m}^{2}$ and is summed up over the course of eight months with a two minute resolution. Several days where omitted due to snow coverage. The results are shown in Fig. 5. The daily energy densities (left axis), shown by the red and green bars, for the SSIM and pyrheliomenter, respectively, are seen to be in excellent agreement across the entire eight month period, differing by only $\pm 1 \%$ on average. Eppley pyrheliometer and SSIM cumulative energy densities for this period are 579.5 and $582.3 \mathrm{kWh} / \mathrm{m}^{2}$, respectively. This difference, of less than $0.5 \%$, indicates truly exceptional agreement, considering the pyrheliometer's measurement uncertainty of $\pm 2 \%$. 


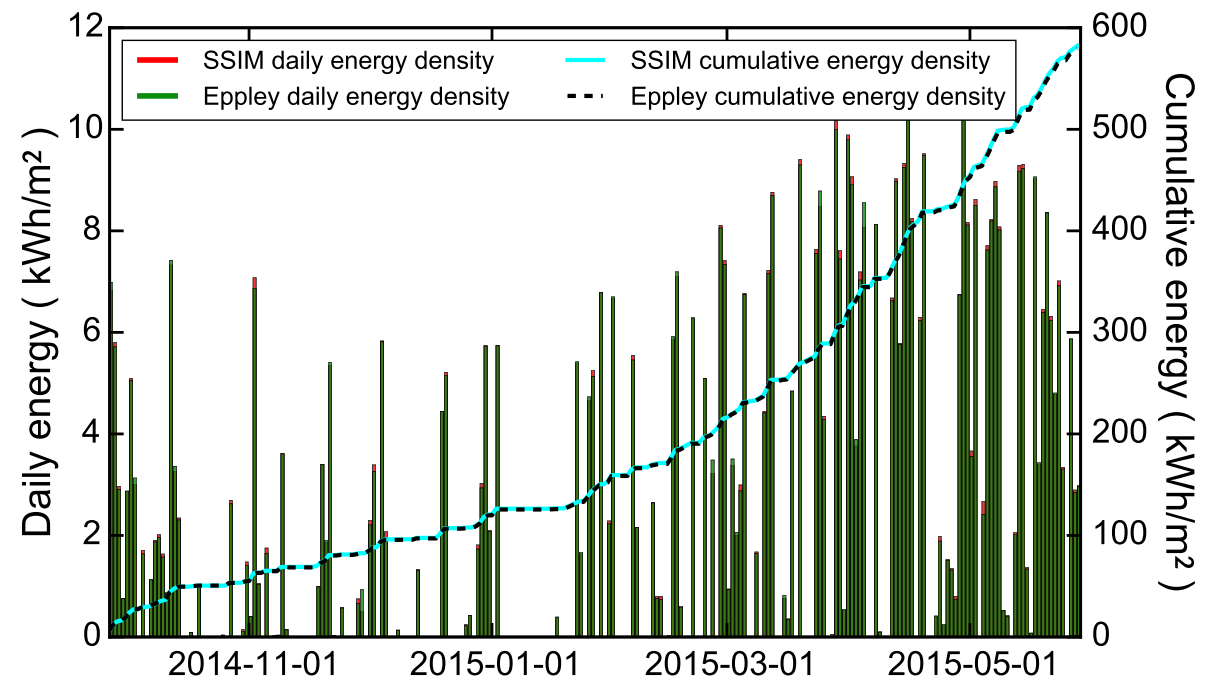

Figure 5: The cumulative and daily energy densities comparison between the Eppley pyrheliometer and the SSIM over eight months. The cumulative energy density as measured by the SSIM agrees to less than $0.5 \%$ of the corresponding value as reported by the Eppley pyrheliometer.

\section{Conclusion}

A SSIM has been presented as a reliable, accurate and cost-effective alternative to a spectroradiometer and a pyrheliometer. Two SSIMs were validated at NREL against secondary reference spectroradiometers and an Eppley ACR. The SSIMs showed an excellent performance against four spectroradiometers at NREL, with a near perfect matching in spectral irradiance under clear sky conditions. The DNI was found to be within $\pm 1 \%$ of the ACR DNI, on average.

SSIM long term performance was validated against a Eppley model NIP pyrheliometer and an ASD spectroradiometer at the SUNLAB Outdoor Test Facility between 27 September 2014 and 28 May 2015. During this period the DNI determined by the SSIM was found to agree within $\pm 1 \%$ of that measured directly by the pyrheliometer with over 20,000 data points used in the analysis. The SSIM was found to accurately reproduce the solar spectrum, with the integrated spectral irradiance agreeing with that of the ASD spectroradiometer and the reference SSIM to within $\pm 1 \%$. Furthermore, the integrated difference in the cumulative energy density between the SSIM and an Eppley pyrheliometer was within $0.5 \%$ over the course of eight months. No degradation of filter bandpass performance or detector sensitivities was observed.

These results demonstrate that the SSIM is an accurate and reliable instrument for measuring solar spectral irradiance in six wavelength bands, providing an accurate reconstruction of the solar spectrum and broadband DNI. The small size, low cost and rugged design with no internal moving components makes it a very strong candidate for routine and dependable monitoring of solar spectral 
irradiance in diverse environments.

\section{Acknowledgment}

The authors would like to acknowledge support from the National Sciences and Engineering Research Council of Canada, the Ontario Research Fund - Research Excellence program, the Ontario Centres of Excellence, Canada Research Chair program, and the Canadian Foundation for Innovation.

The NREL portion of this work was supported by the U.S. Department of Energy under Contract No. DE- AC36-08-GO28308 with the National Renewable Energy Laboratory.

\section{References}

Ångström, A., 1929. On the atmospheric transmission of sun radiation and on dust in the air. Geografiska Annaler 11, 156-166.

Bird, R. E., Riordan, C., 1986. Simple solar spectral model for direct and diffuse irradiance on horizontal and tilted planes at the earth's surface for cloudless atmospheres. Journal of Climate and Applied Meteorology 25 (1), 87-97.

Bodhaine, B. A., Wood, N. B., Dutton, E. G., Slusser, J. R., 1999. On Rayleigh optical depth calculations. Journal of Atmospheric and Oceanic Technology $16,1854-1861$.

Bucholtz, A., 1995. Rayleigh-scattering calculations for the terrestrial atmosphere. Applied Optics 34 (15), 2765-2773.

Cannon, T., Hulstrom, R., 1993. Atmospheric optical calibration system for outdoor testing of photovoltaic devices. Metrologia 30 (4), 409.

Chan, N. L. A., Brindley, H. E., Ekins-Daukes, N. J., 2014. Impact of individual atmospheric parameters on CPV system power, energy yield and cost of energy. Progress in Photovoltaics: Research and Applications 22 (10), 10801095.

Chan, N. L. A., Young, T. B., Brindley, H. E., Ekins-Daukes, N. J., Araki, K., Kemmoku, Y., Yamaguchi, M., 2013. Validation of energy prediction method for a concentrator photovoltaic module in Toyohashi, Japan. Progress in Photovoltaics: Research and Applications 21 (8), 1598-1610.

Fröhlich, C., 1991. History of solar radiometry and the world radiometric reference. Metrologia 28 (3), 111.

García-Domingo, B., Aguilera, J., de la Casa, J., Fuentes, M., 2014. Modelling the influence of atmospheric conditions on the outdoor real performance of a cpv (concentrated photovoltaic) module. Energy 70, 239-250. 
Gilbert, N. L., Goldberg, M. S., Beckerman, B., Brook, J. R., Jerrett, M., 2005. Assessing spatial variability of ambient nitrogen dioxide in montreal, canada, with a land-use regression model. Journal of the Air \& Waste Management Association 55 (8), 1059-1063.

Gueymard, C., 1995. SMARTS2: a simple model of the atmospheric radiative transfer of sunshine: algorithms and performance assessment. Florida Solar Energy Center Cocoa, FL.

Gueymard, C. A., 2001. Parameterized transmittance model for direct beam and circumsolar spectral irradiance. Solar Energy 71, 325-346.

Gueymard, C. A., 2004. The sun's total and spectral irradiance for solar energy applications and solar radiation models. Solar Energy 76, 423-453.

Gueymard, C. A., 2006. SMARTS2 code, version 2.9.5, user's manual. Tech. rep., Solar Consulting Services.

Guter, W., Schöne, J., Philipps, S. P., Steiner, M., Siefer, G., Wekkeli, A., Welser, E., Oliva, E., Bett, A. W., Dimroth, F., 2009. Current-matched triplejunction solar cell reaching $41.1 \%$ conversion efficiency under concentrated sunlight. Applied Physics Letters 94 (22), 223504.

Holben, B., Eck, T., Slutsker, I., Tanre, D., 1998. AERONET - a federated instrument network and data archive for aerosol characterization. Remote Sensing of Environment 66 (1), 1-16.

Holben, B., Tanre, D., Smirnov, A., Eck, T., 2001. An emerging ground-based aerosol climatology: Aerosol optical depth from AERONET. Journal of Geophysical Research: Atmospheres 106 (D11), 12067-12097.

Jacobson, M. Z., 2005. Fundamentals of atmospheric modeling. Cambridge University Press, New York, USA.

McMathon, W., Kurtz, S., Emery, K., Young, M., 2002. Criteria for the design of GalnP/GaAs/Ge triple junction cells to optimize their performance outdoors. Tech. rep., National Renewable Energy Laboratory.

Michalsky, J., Dutton, E. G., Nelson, D., Wendell, J., Wilcox, S., Andreas, A., Gotseff, P., Myers, D., Reda, I., Stoffel, T., et al., 2011. An extensive comparison of commercial pyrheliometers under a wide range of routine observing conditions. Journal of Atmospheric and Oceanic Technology 28 (6), 752-766.

Michalsky, J., Kleckner, E., 1984. Estimation of continuous solar spectral distributions from discrete filter measurements. Solar Energy 33 (1), 57-64.

Muller, M., Marion, B., Kurtz, S., Rodriguez, J., 2010. An investigation into spectral parameters as they impact CPV module performance. In: AIP Conference Proceedings. Vol. 1277. p. 307. 
Osterwald, C., Emery, K., Myers, D., Riordan, C., 1988. Extending the spectral range of silicon-based direct-beam solar spectral radiometric measurements. In: Photovoltaic Specialists Conference, 1988., Conference Record of the Twentieth IEEE. IEEE, pp. 1246-1250.

Press, W. H., Teukolsky, S. A., Vetterling, W. T., Flannery, B. P., 1992. Golden section search in one dimension. Numerical Recipies in C: the Art of Scientific Computing 2.

Reda, I., 1996. Calibration of a solar absolute cavity radiometer with traceability to the world radiometric reference. Tech. rep., National Renewable Energy Lab., Golden, CO, USA.

Reda, I., Andreas, A., 2004. Solar position algorithm for solar radiation applications. Solar energy 76 (5), 577-589.

Schriemer, H., Cook, J. P., Sharma, P., De La Salle, L., Carle, F., White, P. M., Tatsiankou, V., Bosscher, N., Haysom, J. E., Dragomirescu, E., Hinzer, K., 2015. Refractive concentrator optics architectures, tracker precision, and cumulative energy harvest. In: 42nd IEEE Photovoltaic Specialists Conference (PVSC).

Sengupta, M., Habte, A., Kurtz, S., Dobos, A., Wilbert, S., Lorenz, E., Stoffel, T., Renné, D., Gueymard, C. A., Myers, D., et al., 2015. Best practices handbook for the collection and use of solar resource data for solar energy applications. Tech. rep., National Renewable Energy Laboratory.

Tatsiankou, V., 2014. Instrumentation development for site-specific prediction of spectral effects on concentrated photovoltaic system performance. Master's thesis, University of Ottawa.

Tatsiankou, V., Hinzer, K., Muron, A., Haysom, J., Schriemer, H., Myrskog, S., 2014. Solar resource assessment with the solar spectral irradiance meter. In: International Conference on Concentrating Photovoltaic Systems: CPV-10.

Tatsiankou, V., Hinzer, K., Muron, A., Mohammed, J., Wilkins, M., Haysom, J., Schriemer, H., Myrskog, S., 2013. Reconstruction of the solar spectral resource using limited spectral sampling for concentrating photovoltaic systems. In: Photonics North. Vol. 891506. International Society for Optics and Photonics.

Tatsiankou, V., Hinzer, K., Schriemer, H., Haysom, J., Beal, R., 2015. A novel instrument for cost-effective and reliable measurement of solar spectral irradiance. In: Photovoltaic Specialist Conference (PVSC), 2015 IEEE 42nd. IEEE, pp. 1-4.

Wiemer, M., Sabnis, V., Yuen, H., 2011. 43.5\% efficient lattice matched solar cells. In: SPIE Solar Energy+ Technology. International Society for Optics and Photonics, pp. 810804-810804.

WMO, 2008. Guide to meterological instruments and methods of observation. 